\title{
Optimization of production and partial purification of laccase by Phanerochaete chrysosporium using submerged fermenation
}

\author{
Jhadav A. ${ }^{1}$, Vamsi K.K. ${ }^{2}$, Khairnar Y. ${ }^{1}$, Boraste A. ${ }^{3}$, Gupta N. ${ }^{5}$, Trivedi S. ${ }^{4}$, Patil P. ${ }^{6}$, Gupta G. ${ }^{7}$, Gupta M. \\ Mujapara A.K. ${ }^{8}$, Joshi B. ${ }^{9}$, Mishra D. ${ }^{10}$ \\ ${ }^{1}$ Padmashree Dr. D.Y. Patil University, Navi Mumbai, 400614, India \\ ${ }^{2}$ Rai foundations College CBD Belapur Navi Mumbai \\ ${ }^{3}$ S.V.P.M. College of Pharmacy, Baramati, Pune \\ ${ }^{4}$ V.V.P. Engineering College, Rajkot, Gujrat \\ ${ }^{5}$ Sindhu Mahavidyalaya Panchpaoli Nagpur \\ ${ }^{6}$ Dr. D. Y. Patil ACS College, Pimpri, Pune \\ ${ }^{7}$ S.D.S.M. College Palghar, Mumbai \\ ${ }^{8}$ Sir PP Institute of Science, Bhavnagar, Gujrat, adarshmujapara@gmail.com \\ ${ }^{9}$ Rural College of Pharmacy, D.S Road, Bevanahalli, Banglore \\ ${ }^{10}$ Senior Research Coordinator, Reliable analytical laboratory, Thane
}

\begin{abstract}
Laccases are multi copper oxidases having wide substrate specificity mainly found in white-rot fungi, which are the only microorganisms able to degrade the whole wood components. In contrast to most enzymes, which are generally very substrate specific, laccases act on a surprisingly broad range of substrates, including diphenols, polyphenols, different substituted phenols, diamines, aromatic amines, benzenethiols and even some inorganic compounds such as iodine. As they are capable of degrading a wide variety of compounds they are commercially very significant. This project aims at studying the production optimization of laccase using different carbon sources.
\end{abstract}

\section{Introduction}

Proteins that contain copper atoms as cofactors are crucial for numerous reactions in cellular metabolism. They are involved in photosynthesis, oxidative phosphorylation, metal ion homeostasis and catabolism of various nutrients and toxic chemical compounds. Laccase is one such copper protein belonging to the oxidoreductase family [1,2]. When oxidized by a laccase, the reducing substrate loses a single electron and usually forms a free radical. The unstable radical may undergo further laccase-catalysed oxidation or non-enzymatic reactions including hydration, disproportionation and polymerization. Figure no.1 shows the schematic laccase-catalyzed oxidation of a p-diphenol. In laccase-mediated reactions, diphenolic compounds undergo a fourelectron oxidation.
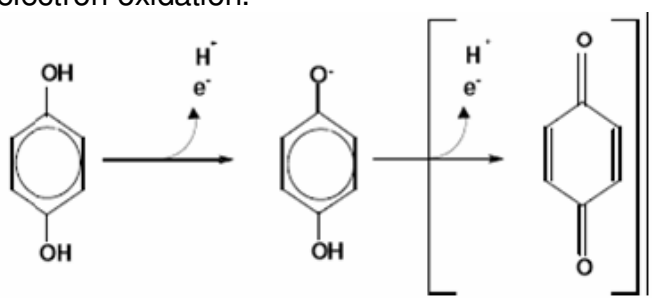

Fig. 1- Laccase-catalyzed oxidation of a diphenol

Laccase is used industrially for pulp delignification, polycyclic aromatic hydrogen degradation, pesticide or insecticide degradation and organic synthesis. With microbial enzymes dominating world markets, more innovation and improvisation is needed to increase the efficiency of production at an economical rate [3]. Laccase is the most widely distributed of all the large blue copper-containing proteins, as it is found in a wide range of higher plants and fungi as well as in bacteria. Laccases in plants have been identified in trees, cabbages, turnips, beets, apples, asparagus, potatoes, pears, and various other vegetables. Laccases have been isolated from Ascomyceteous, Deuteromyceteous and Basidiomyceteous fungi. In the fungi, Ascomycetes and Deuteromycetes have not been a focus for lignin degradation studies as much as the white-rot Basidiomycetes. Laccase from Monocillium indicum was the first laccase to be characterized from an ascomycete showing peroxidative activity. The white-rot basidiomycetes are the most efficient degraders of lignin and also the most widely studied. The enzymes implicated in lignin degradation are: lignin peroxidase, which catalyses the oxidation of both phenolic and non-phenolic units, manganese-dependant peroxidase and laccase, which oxidizes phenolic compounds to give phenoxy radicals and quinines; glucose oxidase and glyoxal oxidase for $\mathrm{H} 2 \mathrm{O} 2$ production and cellobiose-quinone oxidoreductase for quinone reduction. Phanerochaete chrysosporium is a crust fungus, which forms flat fused reproductive fruiting bodies instead of the mushroom structure. This fungi exhibit an interesting pattern of septate hyphae, giving a stronger line of defense in times of distress. The hyphae network has some branching, with diameters ranging from 3-9 $\mu \mathrm{m}$. At the ends of the hyphae rests chlamydospores, thick-walled spores varying from 50-60 $\mu \mathrm{m}$. The conidiophores give rise to round asexual blastoconidia, which are 6-9 $\mu \mathrm{m}$ in diameter. Due to its sustainability at moderate to higher temperatures, specifically $40 \mathrm{oC}$, this white-rot fungus can be found in forests ranging from North America, to areas of Europe and in Iran. Phanerochaete chrysosporium is a saprophytic fungus capable of organic breakdown of the 
woody part of dead plants. Therefore, plants those are in the process of dying or dead serve as an optimal substrate for $P$. chrysosporium. Symptoms may include white patches of cellulose due to the disappearance of lignin from the plant structure. This fungus is not a known pathogen of humans or animals [5]. Laccases of fungi are of particular interest with regard to potential industrial applications, because of their capability to oxidize a wide range of toxic and environmentally problematic substrates. Laccases are currently studied intensively for many applications and they are already used in large scale in the textile industry. Related to textile bleaching, in 1996 Novozyme (Novo Nordisk, Denmark) launched Olaccase enzyme in denim finishing: DeniLite. In 2001, the company Zytex (Zytex Pvt. Ltd. Mumbai, India) developed a formulation based on LMS capable of degrading indigo in a very specific way. The trade name of the product is Zylite. Together with low molecular weight redox-mediator compounds, laccases can generate a desired worn appearance on denim by bleaching indigo dye. The current practical applications of the use of laccase, has led to a search for source of the enzyme from white-rot fungi, which promote or facilitate enzyme action. Indeed, most of the published research and applications of laccase in the forest products industry relate to the pulp and paper sector, where particular emphasis has been placed on studying laccase for use in biobleaching and mill water treatment. Emerging research areas include the tailoring of lignocellulosic materials by laccase-assisted biografting of phenols and other compounds, and the use of laccase for adhesion enhancement in binderless wood boards. Recently, the utility of laccases has also been applied to Nanobiotechnology. This is an increasing research field mainly due to the fact that laccases are able to catalyse electron transfer reactions without additional cofactors and to the development of several techniques for the immobilization of biomolecules such as micropatterning, self-assembled monolayers and layer-by-layer technique. These techniques can be used to immobilize laccases preserving their enzymatic activity [4].

\section{Materials and Methods}

Lyophilized Phanerochaete chrysosporium Strain no. 1197 ATCC 34541 was obtained from National Chemical Laboratories, Pune, India. The culture was maintained in Potato Dextrose Agar

Fungal Staining: Lacto-phenol Cotton Blue was used for staining the fungal culture.

Confirmation of laccase production by Phanerochaete chrysosporium:
Phanerochaete chrysosporium was inoculated on a composite medium containing $0.3 \%$ Peptone, $1 \%$ Glucose, $0.06 \% \mathrm{KH} 2 \mathrm{PO} 4,0.0001 \% \mathrm{ZnSO} 4$, 0.04\% K2HPO4, 0.00005\% FeSO4, 0.005\% MnSO4, 0.05\% MgSO4, 2\% Agar and 0.002\% Guaiacol and observed for color change.

Carbohydrate Source: The primary carbon source used was agricultural wastes which were used in place of Glucose and Guaiacol in a composite medium consisting of $0.1 \% \mathrm{KH} 2 \mathrm{PO} 4$, $0.05 \% \mathrm{MgSO} 4.7 \mathrm{H} 2 \mathrm{O}, 0.02 \% \mathrm{NH} 4 \mathrm{NO} 3,0.001 \%$ $\mathrm{CaCl} 2, \quad 0.0001 \%$ CuSO $4.5 \mathrm{H} 2 \mathrm{O}, \quad 0.0001 \%$ FeSO4.7H2O, $0.0001 \% \quad \mathrm{MnSO} 4$ and $0.01 \%$ Yeast Extract.

Primary Carbon Source:

$\begin{array}{ll}\text { - } & \text { Rice } \\ \text { - } & \text { Wheat } \\ \text { - } & \text { Maize }\end{array}$

The laccase production using the three carbon sources was compared with composite medium containing Glucose only and with glucose and guaiacol which acts as an inducer of laccase.

\section{Assay for laccase activity:}

Table 1- The activity of laccase was measured using Guaiacol by the following protocol

\begin{tabular}{|l|l|l|}
\hline Reagents & Blank & Test \\
\hline $\begin{array}{l}100 \mathrm{mM} \text { Acetate } \\
\text { Buffer }\end{array}$ & $3 \mathrm{ml}$ & $3 \mathrm{ml}$ \\
\hline $10 \mathrm{mM}$ Guaiacol & $1 \mathrm{ml}$ & $1 \mathrm{ml}$ \\
\hline Culture Filtrate & - & $1 \mathrm{ml}$ \\
\hline
\end{tabular}

Absorbance for blank is measured at $470 \mathrm{~nm}$ while that for the test samples were measured at $530 \mathrm{~nm}$. The change in the absorbance of the reaction mixture with guaiacol is monitored for 10 mins of incubation.

Enzyme activity is measured in $\mathrm{U} / \mathrm{ml}$ which is defined as the amount of enzyme catalyzing the production of one micromole of colored product per min per $\mathrm{ml}$.

\section{Calculation:}

$$
\begin{aligned}
& \text { Volume activity }(\mathrm{U} / \mathrm{ml})=\frac{\Delta \mathrm{A}_{430 \mathrm{~m}} \mathrm{~m} / \mathrm{min} \times 4 \mathrm{xVt} \times \text { dilution factor }}{€_{\mathrm{x}} \mathrm{Vs}_{\mathrm{s}}} \\
& \text { Where, } \\
& \mathrm{Vt}=\text { final volume of reaction mixture }(\mathrm{ml})=5.00 \\
& \mathrm{Vs}=\text { sample volume }(\mathrm{ml})=1 \\
& €=\text { extinction co-efficient of guaiacol }=6,740 / \mathrm{M} / \mathrm{cm} \\
& 4=\text { derived from unit definition \& principle }
\end{aligned}
$$

\section{Partial Purification of Laccase}

Crude enzyme was extracted and dialyzed against $20 \mathrm{mM}$ Tris- $\mathrm{HCl}$ buffer $\mathrm{pH}$ 7.5. The dialyzed extract was purified further by ion exchange chromatography using a DEAE column and eluted using $0.1-1.0 \mathrm{M} \mathrm{NaCl}$. The purified sample was analyzed on SDS-PAGE.

\section{Observation and Results Laccase production in Phanerochaete chrysosporium}


$P$. chrysosporium was tested for production of laccase. As seen in Figure no.2, oxidative polymerization of guaiacol was observed in the form of reddish brown product in the medium suggesting laccase activity.

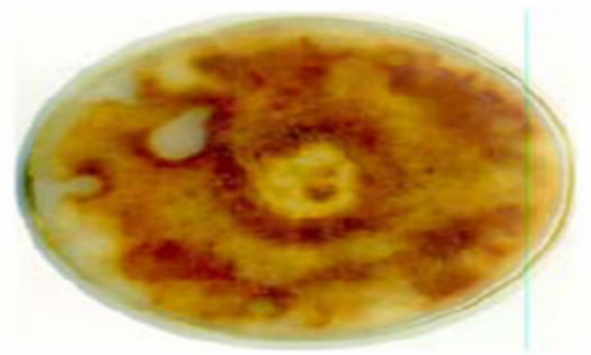

Fig. 2-Oxidative polymerization of guaiacol to form reddish brown zones in the medium

Laccase production optimization with different carbon sources

Laccase production and activity was measured by culturing the fungi in medium containing different carbon sources. Laccase production time was standardized using composite medium containing glucose and guaiacol. Standard time for production of laccase was found to be on the 10 th day (Figure 3 ). Hence 10 days was taken as the standard incubation for production of laccase in medium containing different carbon sources. We observed that there is a slight increase in the activity of laccase when the culture is grown in rice and maize bran containing medium ( 0.24 $\mathrm{U} / \mathrm{ml}$ ) as compared to glucose containing medium $(0.21 \mathrm{U} / \mathrm{ml})$ (Figure 4). We also observed that medium containing glucose in presence of guaiacol gave higher laccase activity as compared to other medium containing glucose, rice bran, wheat bran and maize bran as carbon source (Figure 4).

\section{Purification of laccase}

The laccase from guaiacol medium was dialyzed and purified by ion exchange chromatography. The purified laccase obtained from medium containing glucose and guaiacol showed lower activity than its crude counterpart (Table 1).

To verify the efficiency of the purification, the purified extract was analyzed on $10 \%$ SDSPAGE and stained with Commassie Brilliant Blue. As seen in Figure no.5, laccase was observed adjacent to $66 \mathrm{kD}$ band of molecular weight marker.

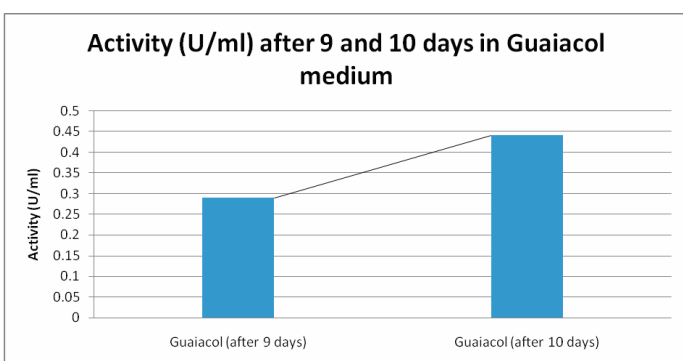

Fig. 3- Graph of laccase activity in medium containing glucose and guaiacol after 9 and 10 days

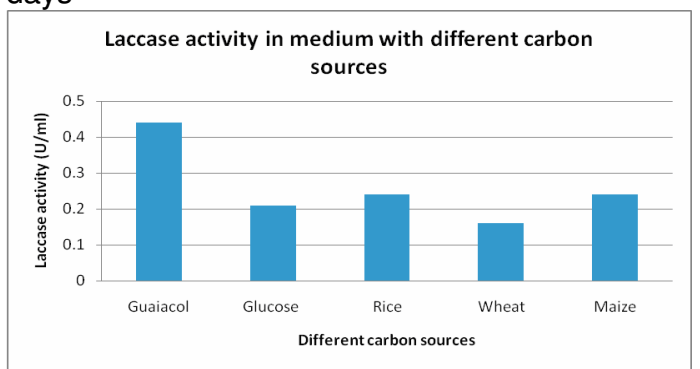

Fig. 4- Laccase activities in medium containing different carbon sources

Table 2- Laccase activities before and after purification of crude enzyme

$\begin{array}{lc}\text { Medium } & \begin{array}{c}\text { Activity } \\ (\mathrm{U} / \mathrm{ml})\end{array} \\ \text { Guaiacol } & 0.44 \\ \text { Purified laccase from } & 0.27 \\ \text { Guaiacol } & \end{array}$

Partial purification of laccase from Guaiacol containing medium

Since the activity of laccase was more in guaiacol containing medium, we tried to partially purify laccase from the medium by ion exchange chromatography (Figure 5). Fractions eluted using $0.8 \mathrm{M} \mathrm{NaCl}$ showed the highest protein content and was hence were analyzed along with crude enzyme by resolving on 10\% SDS PAGE for laccase. 66KDa band was observed on both the crude enzyme and fraction 5 suggesting presence of laccase.

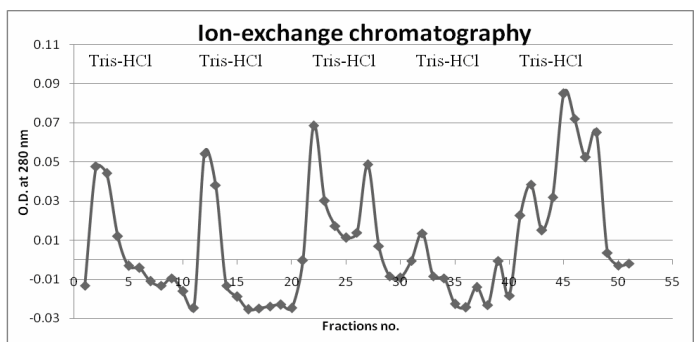

Fig. 5- OD of fractions eluted with increasing salt concentrations in lon exchange chromatography Lane a: molecular marker Lane b: Crude enzyme Lane c: Tris $\mathrm{HCl}+0.8 \mathrm{M} \mathrm{NaCl}$ 
Lane d: Tris $\mathrm{HCl}+0.8 \mathrm{M} \mathrm{NaCl}$

Lane e: Tris $\mathrm{HCl}+0.8 \mathrm{M} \mathrm{NaCl}$

Lane f: Tris $\mathrm{HCl}+0.8 \mathrm{M} \mathrm{NaCl}$

Lane g: Tris $\mathrm{HCl}+0.8 \mathrm{M} \mathrm{NaCl}$

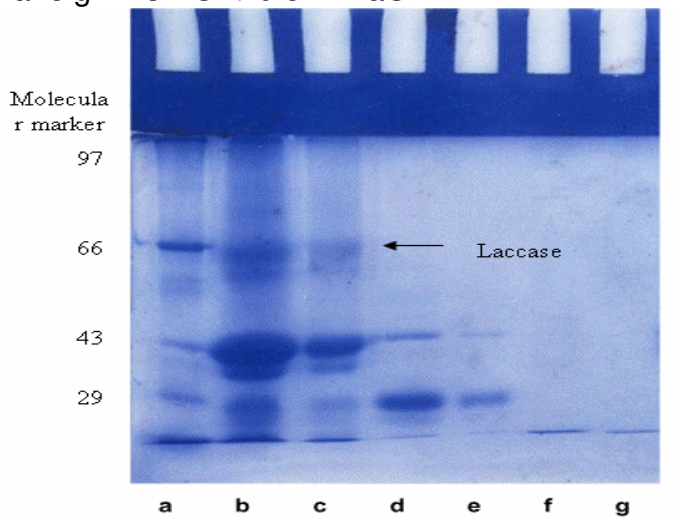

Fig. 6- Fractions eluted using $0.8 \mathrm{M} \mathrm{NaCl}$ were resolved on $10 \%$ SDS-PAGE. Molecular weights of the proteins were compared by loading a molecular weight marker

\section{Discussion}

Laccase is a very unique enzyme capable of degrading multiple substrates. Hence it is no surprise that such an enzyme will be of considerable market value. However, as this enzyme is secreted in very low amounts by the organism a lot of work is being carried out in increasing the production output and optimization. The main objective of our work was to determine if the production efficiency of the enzyme would increase with an alternate carbon source other than glucose. We found a slight increase in the activity when the culture was grown in presence of a rice and maize bran as a carbon source in place of glucose (Figure 4). We also observed in the process that presence of an inducer like guaiacol is required to boost the production (Figure 4). Though we used the inducer in a glucose containing medium we can safely assume that use of inducer in a medium containing alternate carbon source like rice or maize bran would further increase the production of the enzyme. We also determined if the purification process would improve the enzyme recovery and activity and found the activity of the enzyme to decrease. This may be due to the crude purification method which may have resulted in diminishing the enzyme recovery and potency. With better and sophisticated methods of purification we may be able to get good recovery of the enzyme without letting its activity get affected.

\section{References}

[1] Buddolla Viswanath, Subhosh Chandra M. , Pallavi H. and Rajasekhar Reddy B. (2008) African Journal of Biotechnology, 7 (8), 1129-1133.
[2] Kiiskinen, Laura-Leena. Characterization and heterologous production of a novel laccase from Melanocarpus albomyces. ISBN 10: 9513864294 (2004) VTT Publications 556.

[3] Johann Faccelo Osma Cruz (2008) Tarragona : Universitat Rovira i Virgili, 28.arXiv:0801.3337

[4] Buddolla Viswanath, Subhosh Chandra M., Praveen Kumar K., Pallavi H. , Rajasekhar Reddy B. (2008) Dynamic Biochemistry, Process Biotechnology and Molecular Biology, 2(1), 1-13.

[5] Buddolla Viswanath, Subhosh Chandra M., Praveen Kumar K., Rajasekhar Reddy B. (2008) Dynamic Biochemistry, Process Biotechnology and Molecular Biology, 2(1), 19-25. 